\title{
Editorial
}

\author{
José Herskovits · Helder C. Rodrigues • \\ Carlos A. Mota Soares • Claudia Sagastizábal • \\ Argimiro R. Secchi
}

Received: 14 July 2010 / Accepted: 17 August 2010 / Published online: 18 September 2010

(C) Springer Science+Business Media, LLC 2010

This special issue of "OPTE" gathers selected papers in the field of this journal presented at the International Conference on Engineering Optimization, EngOpt 2008, held at Rio de Janeiro on June 2008. The main goal of EngOpt conferences is to periodically bring together engineers, applied mathematicians and computer scientists working on research, development and practical application of optimization methods applied to all engineering disciplines or developing basic techniques.

\footnotetext{
J. Herskovits $(\varangle)$

Mechanical Engineering Program, COPPE-Federal University of Rio de Janeiro, Rio de Janeiro, Brazil

e-mail: jose@optimize.ufrj.br
}

H.C. Rodrigues - C.A. Mota Soares

Instituto Superior Técnico, Technical University of Lisbon, Lisbon, Portugal

H.C. Rodrigues

e-mail: hor@ist.utl.pt

C.A. Mota Soares

e-mail: carlosmotasoares@ dem.ist.utl.pt

C. Sagastizábal

Electric Energy Research Center (CEPEL), Rio de Janeiro, Brazil

e-mail: sagastiz@impa.br

A.R. Secchi

Chemical Engineering Program, COPPE-Federal University of Rio de Janeiro, Rio de Janeiro, Brazil e-mail: arge@peq.coppe.ufrj.br 


\section{Engineering design optimization}

Modern design techniques seek for the best design to perform the desired tasks. Engineering Optimization deals with the optimal design of elements and systems in all engineering fields. Design Optimization techniques are largely employed in most of engineering disciplines, like automotive, aeronautical, mechanical, civil, nuclear, naval, mechanical, electrical, energy and off-shore engineering. This is due to the increase of technological competition and the development of strong and efficient techniques for practical applications.

\section{Energy optimization}

Continuous improvements in computational algorithms and optimization theory have strongly influenced decision making in the energy sector. Today's rapidly evolving energy markets involve critical issues that can only be represented by complex optimization model. Such is the case when devising strategies to reduce carbon emission, and also of designing delivery infrastructures (power transmission lines and gas pipelines), as investments have not kept pace with growing demand and aging equipment. In addition, market reforms replacing traditional, centrally-based planning and control with decentralized, market-based processes and institutions, require special multi-level and stochastic optimization techniques. Finally, the use of enterprise portfolio management, very common in the energy industry, has greatly benefited from state of the art planning models from Stochastic Programming, that usually consider some kind of risk measure as well as financial instruments to hedge the investment portfolio.

\section{Oil \& gas industry}

The growing demand for energy and the increasingly expensive field exploitation, irrigated with sustainability issues, challenge the oil and gas industry to seek innovative ways to increase profitability. In this highly competitive scenario, optimization tools play important roles in many industrial applications, such as, model predictive control, data reconciliation, real-time optimization, heat and mass exchangers, networks design, planning and scheduling. The success of these applications stimulate engineers to go further on more challenging problems, reducing the distance between enterprise-wide driving forces and plant production goals. The multidisciplinary environment of EngOpt forum brings great opportunities for engineers and scientist to share their experiences and look for new ideas to approach these challenges.

\section{Inverse problems}

Numerical methods for inverse problems in most of cases are based on optimization techniques similar to those employed in optimal design. This field, applied in all engineering disciplines, is of utmost importance for Engopt conferences. 


\section{Engineering simulation involving optimization techniques}

Several physical phenomena are naturally represented by an optimization problem. This is the case when the "equilibrium" is attained at the minimum of an energy function. In several applications, constraints must be satisfied. This is the case of contact problems is solids mechanics.

\section{MDO-multidisciplinary design optimization engineering}

Systems are increasingly complex and represented by large and sophisticated numerical models. They involve several interacting disciplines or are made up of distinct interacting subsystems that must be considered simultaneously to obtain efficient designs. Multidisciplinary Design Optimization is devoted to the design of complex systems involving interacting subsystems or disciplines. The main scientific challenges of MDO are concerned with the development of strong and efficient numerical techniques and with the computational organization required for the necessary coupling of codes employed in interacting disciplines.

\section{About interdisciplinarity in engineering optimization}

Modern Engineering Optimization is strongly interdisciplinary in two axes. The need of integration of basic and applied techniques and to solve real engineering problems, requires the cooperation of engineers, mathematicians and computer scientists, working on research and practical application. A fundamental need for MDO is also the establishment of a strong communication of scientists and practitioners acting in different Engineering Disciplines. EngOpt is intended to be a forum to expose and share current and future research and innovation in all techniques involved in Engineering Optimization as well as in the relationships among them.

Guest Editors 